\title{
Anonymous voting and minimal manipulability
}

\author{
Citation for published version (APA):
}

Maus, S., Peters, H. J. M., \& Storcken, A. J. A. (2005). Anonymous voting and minimal manipulability. METEOR, Maastricht University School of Business and Economics. METEOR Research Memorandum No. 012 https://doi.org/10.26481/umamet.2005012

Document status and date:

Published: 01/01/2005

DOI:

10.26481/umamet.2005012

Document Version:

Publisher's PDF, also known as Version of record

\section{Please check the document version of this publication:}

- A submitted manuscript is the version of the article upon submission and before peer-review. There can be important differences between the submitted version and the official published version of record.

People interested in the research are advised to contact the author for the final version of the publication, or visit the DOI to the publisher's website.

- The final author version and the galley proof are versions of the publication after peer review.

- The final published version features the final layout of the paper including the volume, issue and page numbers.

Link to publication

\footnotetext{
General rights rights.

- You may freely distribute the URL identifying the publication in the public portal. please follow below link for the End User Agreement:

www.umlib.nl/taverne-license

Take down policy

If you believe that this document breaches copyright please contact us at:

repository@maastrichtuniversity.nl

providing details and we will investigate your claim.
}

Copyright and moral rights for the publications made accessible in the public portal are retained by the authors and/or other copyright owners and it is a condition of accessing publications that users recognise and abide by the legal requirements associated with these

- Users may download and print one copy of any publication from the public portal for the purpose of private study or research.

- You may not further distribute the material or use it for any profit-making activity or commercial gain

If the publication is distributed under the terms of Article $25 \mathrm{fa}$ of the Dutch Copyright Act, indicated by the "Taverne" license above, 


\title{
Anonymous voting and minimal manipulability
}

\author{
by Stefan Maus*, Hans Peters* and Ton Storcken*
}

March 2005

\begin{abstract}
We compare the manipulability of different choice rules by considering the number of manipulable profiles. We establish the minimal number of such profiles for tops-only, anonymous, and surjective choice rules, and show that this number is attained by unanimity rules with status quo.
\end{abstract}

\section{Introduction}

In choosing new parliamentary representatives most democracies apply voting procedures that select among the top-ranked candidates reported by the voters. It is well known that such procedures are vulnerable to manipulation. For example, if there is an electoral threshold, then votes for a small party might be reconsidered and cast on a (second best) larger party which with high probability will meet the threshold. Also in a district dependent procedure, a voter might opt for the second best if his best candidate has only small support, and in that way prevent a third (worse) candidate to be elected as district representative. In this paper we study voting procedures with respect to this kind of manipulability. Using a natural measure of manipulation, we show that unanimity rules with status quo are the least vulnerable among all reasonable procedures.

We consider a framework in which voting procedures are modelled as choice rules assigning alternatives to profiles of individual preferences. These choice rules are assumed to be tops-only, meaning that they only depend on the topranked alternatives of the voters. Additionally, two standard and natural conditions are imposed: anonymity and unanimity. Anonymity is an egalitarian principle, saying that the names of the voters do not matter. Unanimity is a minimal sovereignty principle: it means that if all voters have the same top candidate, then this candidate is elected. To this setting, however, the well known result of Gibbard [7] and Satterthwaite [20] applies, and therefore any choice rule satisfying the three mentioned conditions is vulnerable to manipulation. This means that, for any such rule, there exist a profile and a voter who, by

*Department of Quantitative Economics, University of Maastricht, P.O. Box 616, 6200 MD Maastricht, The Netherlands. Tel.: +31-43-3883835. E-mail: S.Maus@ke.unimaas.nl, H.Peters@ke.unimaas.nl, T.Storcken@ke.unimaas.nl. 
changing his preference, can induce a new profile resulting in an outcome which is better for him. This kind of manipulation may be undesirable for several reasons. First, the manipulating voter may benefit on the expense of others. Second, in order to obtain a good outcome, the right input should be given to the voting mechanism. Finally, the impossibility of manipulation simplifies the decision process for the voters because they only have to know their own preferences.

There are several strands of research dealing with this manipulability issue. One concerns relaxations of the conditions on the rules at hand. Often, stronger or similar impossibility results are found. See e.g. Pattanaik $[18,19]$ and Ehlers et al. [5]. A second strand of literature is based on a stochastic approach, again often resulting in similar impossibilities. See, e.g., Gibbard [8,9] and Dutta [4]. A third strand imposes preference domain restrictions, often to singlepeaked preferences. If the space of alternatives is one-dimensional, preferences are single-peaked, and the number of voters is odd, then a Condorcet winner exists, which is then a non-manipulable choice. See, e.g., Black [2] or Moulin [17]. If the space of alternatives is more dimensional, then a Condorcet winner usually fails to exist. Depending on the domain of admissible preferences, nonmanipulable choice rules may or may not exist. See, e.g., Kim and Roush [13], Border and Jordan [3], and Zhou [21]. (Of course, the given references are far from constituting a complete list.)

In this paper, we take a different approach. Since all choice rules are manipulable, a natural question is which choice rules are performing best in this respect, i.e., are the least manipulable. To answer this question we need a measure of manipulability. An intuitive measure is to count the number of profiles at which a given choice rule is manipulable: the larger this number the more manipulable the choice rule is. This measure was introduced by Kelly [10]. He found the minimal number of manipulable profiles for choice rules which are unanimous and non-dictatorial in the case of two agents ${ }^{1}$ and three alternatives. See also Kelly $[11,12]$. In Fristrup and Keiding [6] this minimal number was found for an arbitrary number of alternatives and two agents. Maus et al. [14] obtain a general result for arbitrary numbers of agents and alternatives: almost dictatorial rules are the least vulnerable to manipulation among all non-dictatorial and unanimous rules. In Maus et al. [15] the minimal degree of manipulation for surjective and anonymous choice rules is determined. In Maus et al. [16] this degree is found for unanimous and anonymous choice rules for the case of three alternatives and an arbitrary number of agents. By enumeration and simulation techniques, Aleskerov and Kurbanov [1] determine the minimal number of manipulable profiles for twenty six well-known choice rules such as Borda and plurality. They also discuss other measures for manipulation.

The present paper is different since we confine ourselves to tops-only choice rules - often called voting rules. We show that among all unanimous and anonymous voting rules, the unanimity rule with status quo is doing best with respect to manipulability. This rule chooses a given fixed alternative (the status quo)

\footnotetext{
${ }^{1}$ In general we use the term "agent" rather than "voter".
} 
unless all voters have the same best alternative, possibly different from the status quo. We derive this result under the assumption that the number of agents exceeds the number of alternatives. The fraction of manipulable profiles for this rule turns out to be of order $n \cdot m^{2-n}$, where $n$ is the number of voters and $m$ the number of candidates. So this rule is among the few choice rules which are not highly manipulable (cf. Kelly [12]). Clearly, this choice rule is only occasionally used, for instance in the Council of the European Union, and its rigidity makes it hardly applicable in elections. Therefore, the result presented here is an exploring step setting an absolute lower bound on the measure of manipulation in voting rules, rather than a recommendation to use unanimity with status quo rules. Moreover, we do not know if it holds true if manipulation is measured differently (see Aleskerov and Kurbanov [1]). On the other hand, this lower bound makes it possible to compare the level of manipulation of a given rule to what is achievable in this respect.

Our proof of this result is based on combinatorial arguments which have no bite if the number of agents does not exceed the number of alternatives. For the latter case some partial results are mentioned in the final section and in an appendix to the paper. It turns out that for two agents unanimity rules with status quo are not necessarily minimally manipulable. Also for two agents, we obtain a characterization of all minimally manipulable rules under the stronger condition of Pareto optimality instead of unanimity.

The paper is organized as follows. Section 2 contains preliminaries and introduces unanimity rules with status quo. Section 3 considers the case where the number of alternatives is at least four, and Section 4 extends this to three alternatives. Section 5 concludes.

\section{Unanimity rules with status quo}

Throughout we consider a finite set $A$ of $m$ alternatives and a set $N=\{1,2, \ldots, n\}$ of agents. Unless stated otherwise we assume $n>2$. The agents have linear preferences over the alternatives, i.e. (strongly) complete, antisymmetric and transitive relations on $A$. Let $L(A)$ denote the set of all these preferences. A choice rule is a function $f$ from $L(A)^{N}$ to $A$, where $L(A)^{N}$ denotes the set of profiles $p$ of linear orderings. At a profile $p$ the preference of agent $i \in N$ is denoted by $p(i)$. Let $a, b$ and $c$ be three alternatives in $A$. Then $\ldots a \ldots b \ldots=p(i)$ means that $a$ is strictly preferred to $b$ at $p(i)$ and $c \ldots=p(i)$ means that $c$ is best at $p(i)$; in that case we also write $\operatorname{top}(p(i))=c$. For a profile $p$ in $L(A)^{N}$ the function $\operatorname{top}(p)$ in $A^{N}$ is defined by $\operatorname{top}(p)(i)=\operatorname{top}(p(i))$ for all agents $i \in N$. Also, $\operatorname{topset}(p)=\{\operatorname{top}(p(i)): i \in N\}$ is the set of alternatives that are at least once at the top of an agent's preference in $p$. For a profile $p \in L(A)^{N}$ and an alternative $a$ in $A$ let $N(a, p)=\{i \in N: \operatorname{top}(p(i))=a\}$ and $n(a, p)=|N(a, p)|$, where $|S|$ denotes the cardinality of the set $S$.

A choice rule $f$ is called anonymous if it is symmetric in its arguments. It is called surjective if (as usual) $f\left(L(A)^{N}\right)=A$. Here, for all $V \subseteq L(A)^{N}$, the image of $V$ under $f$ is denoted by $f(V)$. A slightly stronger condition than 
surjectivity is unanimity: this means that for profiles $p$, if $\operatorname{topset}(p)=\{a\}$ for some alternative $a$, then $f(p)=a$. So, if all agents order alternative $a$ best, then it is chosen. A choice rule $f$ is called tops-only if $f(p)=f(q)$ for all profiles $p$ and $q$ with $\operatorname{top}(p)=\operatorname{top}(q)$. So the outcome of a tops-only choice rule at a profile is completely determined by the best alternatives of the agents: such a rule is usually called a voting rule.

For an agent $i \in N$, profiles $p$ and $q$ are $i$-deviations if $p(j)=q(j)$ for all $j \neq i$. A choice rule $f$ is manipulable (at profile $p$ by agent $i$ towards profile $q)$ if $p$ and $q$ are $i$-deviations, $f(p) \neq f(q)$ and $\ldots f(q) \ldots f(p) \ldots=p(i)$. In such a case agent $i$ can benefit at profile $p$ by reporting $q(i)$ in stead of $p(i)$. Let $M_{f}$ denote the set of all profiles at which choice rule $f$ is manipulable. Then $\left|M_{f}\right|$ measures the manipulability of choice rule $f$. If $\left|M_{f}\right|$ is equal to zero, then at every profile the choice rule is not manipulable, in which case it is said to be strategy-proof. If there are at least three alternatives, then only dictatorial rules are strategy-proof and surjective: this is the well-known result of Gibbard (1973) and Satterthwaite (1975). Let $F$ denote the class of all anonymous, surjective and tops-only choice rules and let $m_{F}=\min \left\{\left|M_{f}\right|: f \in F\right\}$. So $m_{F}$ is the minimal level of manipulability among the choice rules in $F$. Because dictatorial rules are tops-only but not anonymous, $m_{F}$ is strictly larger than zero.

For an alternative $a$ we define the unanimity rule with status quo a, denoted by $u_{a}$, as follows. Let $p$ be a profile. Then $u_{a}(p):=x$ if $\{x\}=\operatorname{topset}(p)$ for some $x \in A$, and $u_{a}(p)=a$ in all other cases. So an alternative $x$ different from $a$ is chosen only if all agents consider it best. The main result of this paper is that unanimity rules with status quo are the minimally manipulable rules among all anonymous surjective rules, provided $n>m \geq 3$. The number of manipulable profiles $\left|M_{u_{a}}\right|$ can be computed as follows. Consider a profile $p \in M_{u_{a}}$. Then for some agent $i$ and some $i$-deviation $q, u_{a}(p) \neq u_{a}(q)$ and $\ldots u_{a}(q) \ldots u_{a}(p) \ldots=p(i)$. Clearly $u_{a}(p)=a$ and $u_{a}(q) \neq a$. So, $u_{a}(q) \ldots=p(j)$ for all agents $j \in N-\{i\}$. As $u_{a}(p) \neq u_{a}(q)$ it follows that $\operatorname{top}(p(i)) \neq u_{a}(q)$. Since there are $\frac{m !}{2}$ preferences $p(i)$ with $u_{a}(q)$ ranked above $a$ but $(m-1)$ ! of these have $u_{a}(q)$ on top, it follows that there are $\frac{m !}{2}-(m-1)$ ! preferences $p(i)$ which result in a manipulable profile. Since we can choose $i$ from a set of $n$ agents, $u_{a}(q) \neq a$ from $m-1$ alternatives, and the other alternatives can be ordered by the other agents in $((m-1) !)^{n-1}$ ways, we find altogether that

$$
\begin{aligned}
\left|M_{u_{a}}\right| & =n \cdot(m-1) \cdot\left(\frac{m !}{2}-(m-1) !\right) \cdot((m-1) !)^{(n-1)} \\
& =\frac{1}{2} n(m-1)(m-2)((m-1) !)^{n}
\end{aligned}
$$

We end this section with a combinatorial observation which is used extensively in the following two sections.

Remark 1 Let $m \geqslant 3$ and let $p$ be a profile with topset $(p)=\left\{x_{1}, x_{2}, \ldots, x_{k}\right\}$. Let the anonymous and tops-only choice rule $f$ be manipulable at profile $p$ by 
agent $i \in N\left(x_{1}, p\right)$ towards profile $q$. Then, obviously, $f(p) \neq x_{1}$. There are

$$
\frac{n !}{n\left(x_{1}, p\right) ! \cdot n\left(x_{2}, p\right) ! \ldots n\left(x_{k}, p\right) !}((m-1) !)^{n}
$$

profiles $r$ which by anonymity and tops-onliness yield the same outcome as $p$ under $f$. As $f(p) \neq x_{1}$, at most

$$
\frac{n !}{n\left(x_{1}, p\right) ! \cdot n\left(x_{2}, p\right) ! \ldots n\left(x_{k}, p\right) !}((m-1) !)^{n-n\left(x_{1}, p\right)} \cdot\left(\frac{(m-1) !}{2}\right)^{n\left(x_{1}, p\right)}
$$

of these profiles are such that all agents in $N\left(x_{1}, p\right)$ prefer $f(p)$ to $f(q)$, and therefore are not manipulable by such an agent from $p$ to $q$. Subtracting (3) from (2), we obtain

$$
\left|M_{f}\right| \geqslant \frac{n !}{n\left(x_{1}, p\right) ! \cdot n\left(x_{2}, p\right) ! \ldots n\left(x_{k}, p\right) !}((m-1) !)^{n}\left(1-\left(\frac{1}{2}\right)^{n\left(x_{1}, p\right)}\right) .
$$

\section{Minimal manipulation with four or more al- ternatives}

In this section we prove that among all surjective, anonymous and tops-only choice rules only unanimity rules with status quo are minimally manipulable, provided that $n>m \geqslant 4$. In the next section we extend this result to $n>m \geqslant$ 3.

Let $f \in F$ such that $\left|M_{f}\right| \leqslant\left|M_{u_{a}}\right|$. The proof of the announced result runs as follows. For $1 \leqslant k \leqslant m$ let $B_{k}=\left\{p \in L(A)^{N}:|\operatorname{topset}(p)| \geqslant k\right\}$. So $B_{k}$ is the set of profiles at which there are at least $k$ different top alternatives. We first show that, if $k \geqslant 4$, then $B_{k}$ is disjoint from $M_{f}$ and, moreover, that $f$ is constant on $B_{4}$. Next, we successively show that $f$ is constant on $B_{3}$ and $B_{2}$. Finally, we show that $f$ is a unanimity rule with status quo.

Lemma 1 Let $n>m \geqslant 4$ and $p \in B_{4}$. Then $p \notin M_{f}$.

Proof. Suppose that $f$ is manipulable at $p$. By (4) there is an alternative $x_{1}$ such that

$$
\begin{aligned}
\left|M_{f}\right| & \geqslant \frac{n !}{n\left(x_{1}, p\right) ! \cdot n\left(x_{2}, p\right) ! \ldots n\left(x_{4}, p\right) !}((m-1) !)^{n}\left(1-\left(\frac{1}{2}\right)^{n\left(x_{1}, p\right)}\right) \\
& \geqslant \frac{n !}{(n-3) !} \cdot \frac{1}{2} \cdot((m-1) !)^{n} \\
& >\left|M_{u_{a}}\right|
\end{aligned}
$$

where the last inequality follows by (1). This is a contradiction, which completes the proof. 
Lemma 2 Let $n>m \geqslant 3$ and let $k \geqslant 3$. Suppose $B_{k} \cap M_{f}=\varnothing$. Then there is an alternative $a$ such that $f\left(B_{k}\right)=\{a\}$.

Proof. Let $p, q \in B_{k}$ and $i \in N$ such that $p$ and $q$ are $i$-deviations. It is sufficient to prove that $f(p)=f(q)$. To the contrary assume that $f(p)=a \neq$ $b=f(q)$. As neither $p$ nor $q$ are in $M_{f}$ it follows that $\ldots f(p) \ldots f(q) \ldots=p(i)$ and $\ldots f(q) \ldots f(p) \ldots=q(i)$.

Suppose $\operatorname{top}(p(i))=c \neq f(p)$. Then for an $i$-deviation $r$ of $p$ such that $r(i)=c \ldots f(q) \ldots f(p) \ldots$ we would have, by tops-onliness: $f(r)=f(p)$, hence $i$ could manipulate from $r$ towards $q$. Since $r \in B_{k}$, this contradicts $B_{k} \cap M_{f}=\varnothing$. Hence $\operatorname{top}(p(i))=f(p)=a$. Similarly it follows that $\operatorname{top}(q(i))=f(q)=b$. So, $n(a, p)=n(a, q)+1$ and $n(b, p)+1=n(b, q)$. Since $p$ and $q$ are $i$-deviations in $B_{k}$ and $k \geqslant 3$, there is an alternative $c \in A-\{a, b\}$ and an individual $j \in N(c, p) \cap N(c, q)$. Consider profiles $v$ and $w$ such that $v$ is a $j$-deviation of $p$ with $b \ldots=v(j)$ and $w$ satisfies $v(i)=w(j), v(j)=w(i)$, and $v(l)=w(l)$ for all $l \neq i, j$. Note that $q$ and $w$ are $j$-deviations. Suppose $f(v) \neq a$. Then by tops-onliness we may assume without loss of generality that $\ldots f(v) \ldots a \ldots=p(j)$. But then $f$ is manipulable at $p$ by $j$ towards $v$, a contradiction since $p \in B_{k}$. So $f(v)=a$. Then, by anonymity, $f(w)=a$. Because of tops-onliness we may assume without loss of generality that ...a...b... $=q(j)$. This makes $f$ manipulable at $q$ by $j$ towards $w$, which yields a contradiction with $q \in B_{k}$. Hence, $f(p)=f(q)$.

Lemma 3 Let $n>m \geqslant 4$. There is an alternative a such that $f\left(B_{3}\right)=\{a\}$.

Proof. Lemma 1 implies that $M_{f} \cap B_{4}=\emptyset$. So Lemma 2 implies that there is an alternative $a \in A$ such that $f\left(B_{4}\right)=\{a\}$. Let $p \in B_{3}-B_{4}$. It is sufficient to prove that $f(p)=a$. To the contrary suppose $f(p) \neq a$. Since $p \in B_{3}-B_{4}$ it follows that $|\operatorname{topset}(p)|=3$, say topset $(p)=\left\{x_{1}, x_{2}, x_{3}\right\}$.

First we show that $A \subseteq\left\{x_{1}, x_{2}, x_{3}, a\right\}$. To the contrary suppose that $b \in$ $A-\left\{x_{1}, x_{2}, x_{3}, a\right\}$. Since $n>m \geqslant 4$ we may without loss of generality assume that $n\left(x_{1}, p\right) \geqslant 2$. Let $i \in N\left(x_{1}, p\right)$ and consider an $i$-deviation $q$ from $p$ such that $b \ldots=q(i)$ and $\ldots f(p) \ldots a \ldots=q(i)$. Since $q \in B_{4}, f(q)=a$. As $f$ is manipulable at profile $q$ by $i$ towards $p$ we have a contradiction with Lemma 1 . Hence, $A \subseteq\left\{x_{1}, x_{2}, x_{3}, a\right\}$. In particular, $m=4$ and $a \notin\left\{x_{1}, x_{2}, x_{3}\right\}$. We have also proved that $f(r)=a$ for any profile $r \in B_{3}-B_{4}$ such that $a \in \operatorname{topset}(r)$.

Since $f(p) \neq a$, by tops-onliness we may assume without loss of generality that $f(p)=x_{1}$ and $\ldots a \ldots f(p) \ldots=p(i)$ for some $i \in N\left(x_{2}, p\right) \cup N\left(x_{3}, p\right)$. Consider an $i$-deviation $q$ of $p$ with $a \ldots=q(i)$. We claim that $f(q)=a$. Indeed, if $q \in B_{4}$ then this follows from $f\left(B_{4}\right)=\{a\}$, and if $q \in B_{3}-B_{4}$ this follows from the observation in the last sentence of the previous paragraph. But now, $f$ is manipulable at $p$ by $i$ towards $q$. Thus, by applying Remark 1 to $p$ for an agent $i$ in $N\left(x_{2}, p\right)$ and also for an agent $i$ in $N\left(x_{3}, p\right)$ we obtain

$$
\left|M_{f}\right| \geqslant \frac{n !}{n\left(x_{1}, p\right) ! n\left(x_{2}, p\right) ! n\left(x_{3}, p\right) !} \cdot((m-1) !)^{n} \cdot\left(1-\left(\frac{1}{2}\right)^{n\left(x_{2}, p\right)}\right)
$$




$$
\begin{aligned}
& +\frac{n !}{n\left(x_{1}, p\right) ! n\left(x_{2}, p\right) ! n\left(x_{3}, p\right) !} \cdot((m-1) !)^{n} \cdot\left(1-\left(\frac{1}{2}\right)^{n\left(x_{3}, p\right)}\right) \\
\geqslant & \frac{n !}{n\left(x_{1}, p\right) ! n\left(x_{2}, p\right) ! n\left(x_{3}, p\right) !} \cdot((m-1) !)^{n} .
\end{aligned}
$$

Hence

$$
\frac{\left|M_{f}\right|}{\left|M_{u_{a}}\right|} \geqslant \frac{n !}{(n-2) !} \cdot \frac{2}{n(m-1)(m-2)}=\frac{(n-1)}{3}>1,
$$

a contradiction. So $f(p)=a$ and the proof is complete.

Lemma 4 Let $n>m \geqslant 4$ and let $f\left(B_{3}\right)=\{a\}$ for some $a \in A$. Then $f\left(B_{2}\right)=$ $\{a\}$.

Proof. Let $p \in B_{2}-B_{3}$. It is sufficient to prove that $f(p)=a$. Let $x$ and $y$ be two alternatives and $S$ and $T$ be two non-empty subsets of $N$, such that $S=N(x, p), T=N(y, p)$ and $S \cup T=N$. Let $s=|S|$ and $t=|T|$, such that $s \geqslant t$. Suppose $f(p) \neq a$.

First suppose that $t \geqslant 2$. Consider profiles $q \in B_{3}$ which are $i$-deviations of $p$ for some $i$ in $N$ such that $z \ldots=q(i)$ for some alternative $z \in A-\{x, y, a\}$ and $\ldots f(p) \ldots a \ldots=q(i)$. Because of tops-onliness we may assume that for some $j$ in $N$ we have $\ldots a \ldots f(p) \ldots=p(j)$, where $j \in S$ if $f(p) \neq x$ and $j \in T$ if $f(p) \neq y$. So, since $f(q)=a$ it follows that $f$ is manipulable both at $p$ by $j$ towards $q$ and at $q$ by $i$ towards $p$. So, by applying Remark 1 to profiles $q$ and $p$ we have

$$
\begin{aligned}
\left|M_{f}\right| \geqslant & (m-3) \cdot \frac{n !}{(s-1) ! t !} \cdot((m-1) !)^{n} \cdot\left(1-\left(\frac{1}{2}\right)^{1}\right) \\
& +(m-3) \cdot \frac{n !}{s !(t-1) !} \cdot((m-1) !)^{n} \cdot\left(1-\left(\frac{1}{2}\right)^{1}\right) \\
& +\frac{n !}{s ! t !} \cdot((m-1) !)^{n} \cdot\left(1-\left(\frac{1}{2}\right)^{t}\right) \\
= & (m-3) \cdot\left(\left(\frac{n !}{(s-1) ! t !}+\frac{n !}{s !(t-1) !}\right) \cdot \frac{1}{2}\right. \\
& \left.+\frac{n !}{s ! t !} \cdot\left(1-\left(\frac{1}{2}\right)^{t}\right)\right) \cdot((m-1) !)^{n} \\
= & (m-3) \cdot\left(\frac{n \cdot n !}{s ! t !} \cdot \frac{1}{2}+\frac{n !}{s ! t !} \cdot\left(1-\left(\frac{1}{2}\right)^{t}\right)\right) \cdot((m-1) !)^{n} .
\end{aligned}
$$

Here, the first two terms after the inequality sign relate to manipulations at $q$ towards $p$, and the last term to manipulations at $p$ towards $q$. From (5), as $t \geqslant 2$,

$$
\left|M_{f}\right|>\left((m-3) \cdot \frac{n \cdot n !}{2 \cdot s ! t !}+\frac{n !}{2 \cdot s ! t !}\right) \cdot((m-1) !)^{n}
$$


and

$$
\begin{aligned}
\frac{\left|M_{f}\right|}{\left|M_{u_{a}}\right|} & >\frac{((m-3) n+1) \cdot n !}{2 \cdot s ! t !} \cdot \frac{2}{n(m-1)(m-2)} \\
& \geqslant \frac{((m-3) n+1)(n-1) !}{2 !(n-2) !(m-1)(m-2)} \\
& \geqslant \frac{((m-3) n+1)(n-1)}{2(m-1)(m-2)} \\
& \geqslant \frac{((m-3)(m+1)+1) m}{2(m-1)(m-2)} \\
& >1,
\end{aligned}
$$

where the last inequality follows since $m \geq 4$. This contradicts our assumption $\left|M_{f}\right| \leqslant\left|M_{u_{a}}\right|$. Hence, $f(p)=a$ if $t \geqslant 2$.

Now let $t=1$. Consider $i$-deviations $q$ for $i \in S$ such $z \ldots=q(i)$ for $z \in$ $A-\{a, x\}$ and $\ldots f(p) \ldots a \ldots=q(i)$. Because $q \in B_{3}$ in case $z \neq y$ or $n(z, q)=2$ in case $z=y$, we have $f(q)=a$ and therefore that $f$ is manipulable at $q$. Hence, by applying Remark 1 to profiles $q$ to cases where $z \neq y$ and to cases where $z=y$ we have that

$$
\begin{aligned}
\left|M_{f}\right| \geqslant & (m-3) \cdot \frac{n !}{(n-2) !} \cdot\left(1-\left(\frac{1}{2}\right)^{1}\right) \cdot((m-1) !)^{n} \\
& +\frac{n !}{(n-2) ! 2} \cdot\left(1-\left(\frac{1}{2}\right)^{2}\right) \cdot((m-1) !)^{n} \\
= & \left((m-3) \frac{1}{2}+\frac{3}{8}\right) \cdot \frac{n !}{(n-2) !} \cdot((m-1) !)^{n} .
\end{aligned}
$$

Hence

$$
\begin{aligned}
& \frac{\left|M_{f}\right|}{\left|M_{u_{a}}\right|} \geqslant \frac{\left((m-3) \frac{1}{2}+\frac{3}{8}\right) \cdot \frac{n !}{(n-2) !}}{\frac{1}{2} \cdot n \cdot(m-1) \cdot(m-2)} \\
&=\frac{(m-3)(n-1)+\frac{3}{4}(n-1)}{(m-1)(m-2)} \\
& \geqslant \frac{m\left(m-2 \frac{1}{4}\right)}{(m-1)(m-2)} \\
&>1,
\end{aligned}
$$

where the last inequality follows since $m \geqslant 4$. This contradicts our assumption $\left|M_{f}\right| \leqslant\left|M_{u_{a}}\right|$ and therefore completes the proof.

The following theorem shows that in case of at least four alternatives minimal manipulation is only satisfied by unanimity rules with status quo. Recall that $F$ denotes the set of all surjective, tops-only and anonymous choice rules.

Theorem 1 Let $n>m \geqslant 4$. Let $f \in F$. Then $\left|M_{f}\right| \leqslant\left|M_{g}\right|$ for all $g \in F$ if and only if $f$ is a unanimity rule with status quo. 
Proof. Assume that $\left|M_{f}\right| \leqslant\left|M_{g}\right|$ for all $g \in F$. It is sufficient to show that $f$ is a unanimity rule with status quo. By Lemmas 3 and 4 there is an alternative $a \in A$ such that $f\left(B_{2}\right)=\{a\}$. For every $x \in A$ let $p_{x}$ denote a profile such that topset $\left(p_{x}\right)=\{x\}$. By tops-onliness it is sufficient to prove that $f\left(p_{x}\right)=x$, for then $f=u_{a}$, the unanimity rule with status quo $a$. Let

$$
\begin{aligned}
& A_{1}=\left\{x \in A-\{a\}: f\left(p_{x}\right)=x\right\}, \\
& A_{2}=\left\{x \in A-\{a\}: f\left(p_{x}\right)=y \text { for some } y \notin\{x, a\}\right\}, \\
& A_{3}=\left\{x \in A-\{a\}: f\left(p_{x}\right)=a\right\}, \text { and } \\
& A_{4}=\left\{x \in A-\{a\}: f\left(p_{a}\right)=x\right\} .
\end{aligned}
$$

Let $m_{i}=\left|A_{i}\right|$ for $i \in\{1,2,3,4\}$. Then $m_{4} \in\{0,1\}$ and, by surjectivity, $m_{3} \leqslant 1$ and $m_{3}=1 \Rightarrow m_{4}=1$. Hence, $m_{4} \geqslant m_{3}$ and since $m_{1}+m_{2}+m_{3}=m-1$, we have

$$
m_{1}+m_{2}+m_{4} \geqslant m-1 \text {. }
$$

By a similar argument as the one resulting in (1), there are exactly $\frac{1}{2}(m-$ 2) $((m-1) !)^{n}$ manipulable profiles for each $x \in A_{1}$, hence in total

$$
m_{1} \cdot \frac{1}{2}(m-2)((m-1) !)^{n}
$$

Now consider $x \in A_{2}$. The total number of profiles of the format $p_{x}$ is equal to $((m-1) !)^{n}$. These profiles are manipulable unless $f\left(p_{x}\right)$ is ranked above $a$ for each agent (since $\left.f\left(B_{2}\right)=\{a\}\right)$. This results in $((m-1) !)^{n}-((m-1) ! / 2)^{n}=$ $((m-1) !)^{n} \cdot\left(1-(1 / 2)^{n}\right)$ manipulable profiles. Furthermore, if $q$ is an $i$-deviation of $p_{x}$ such that $\ldots f\left(p_{x}\right) \ldots a \ldots=q(i)$ and $x \ldots \neq q(i)$, then $f$ is manipulable at $q$ by $i$ towards $p_{x}$ since $f(q)=a$. This results in another $n \cdot(m !-(m-1) !) / 2$. $((m-1) !)^{n-1}$ manipulable profiles, namely all such deviations with $x$ not on top for exactly one agent and $f\left(p_{x}\right)$ ranked above $a$ for the same agent. In total, this adds

$$
m_{2} \cdot\left(\left(1-\left(\frac{1}{2}\right)^{n}\right)+\frac{1}{2} n(n-1)\right)((m-1) !)^{n}
$$

manipulable profiles.

Next, consider $x \in A_{4}$, hence $x=f_{p}(a)$ and $x \neq a$. Consider an $i$-deviation $q$ of $p_{a}$ such that $\ldots a \ldots f\left(p_{a}\right) \ldots=q(i)$ and $\ldots a \neq q(i)$. Then, since $f(q)=a, f$ is manipulable at $p_{a}$ towards $q$. This yields $((m-1) !)^{n}$ manipulable profiles, namely all profiles of the format $p_{a}$. On the other hand, for an $i$-deviation $q$ of $p_{a}$ with $\ldots f\left(p_{a}\right) \ldots a \ldots=q(i)$ we have that $f$ is manipulable by $i$ at $q$ towards $p_{a}$. Since there are $m ! / 2$ preferences where $f\left(p_{a}\right)$ is ranked above $a$, this results in another $(m ! / 2) \cdot n \cdot((m-1) !)^{n-1}=\frac{1}{2} n m((m-1) !)^{n}$ manipulable profiles. So to the total this adds

$$
m_{4} \cdot\left(\frac{1}{2} n m+1\right)((m-1) !)^{n}
$$

manipulable profiles. Combining (1) with (7)-(9), we obtain

$$
\frac{1}{2}(m-1)(m-2)((m-1) !)^{n} \geqslant\left|M_{f}\right|
$$




$$
\begin{aligned}
\geqslant \quad & m_{1} \cdot \frac{1}{2}(m-2)((m-1) !)^{n} \\
& +m_{2}\left(\left(1-\left(\frac{1}{2}\right)^{n}\right)+\frac{1}{2} n(n-1)\right)((m-1) !)^{n} \\
& +m_{4}\left(\frac{1}{2} n m+1\right)((m-1) !)^{n} .
\end{aligned}
$$

If $m_{2} \neq 0$ or $m_{4} \neq 0$ then the right-hand side of (10) is strictly larger than

$$
\begin{array}{r}
\frac{1}{2}((m-1) !)^{n} \cdot\left[m_{1}(m-2)+m_{2}(m-2)+m_{4}(m-2)\right] \\
\geqslant \frac{1}{2} n(m-1)(m-2)((m-1) !)^{n}
\end{array}
$$

where we use (6) for the last inequality. This contradicts (10), hence $m_{2}=$ $m_{4}=m_{3}=0$ and $m_{1}=m-1$. Thus, $f\left(p_{x}\right)=x$ for all $x \in A$. This completes the proof.

\section{Minimal manipulation with three alternatives}

In this section the result of the previous section is extended to the three alternatives case. We show that among all surjective, anonymous and tops-only choice rules only unanimity rules with status quo are minimally manipulable. For the rest of this section let $n>m=3$ and let $f \in F$ with $\left|M_{f}\right| \leqslant\left|M_{u_{a}}\right|$. We first show that $f$ is not manipulable at a profile with three different top alternatives. Lemma 2 then implies that $f$ is constant on $B_{3}$. Next, this is established for $B_{2}$. Then, similar to Theorem 1 , it follows that $f$ is a unanimity rule with status quo.

Lemma 5 Let $p \in B_{3}$. Then $p \notin M_{f}$.

Proof. Suppose that $f$ were manipulable at $p$ by some agent, say $i$ in $N\left(x_{1}, p\right)$. Remark 1 then implies that

$$
\left|M_{f}\right| \geqslant \frac{n !}{n\left(x_{1}, p\right) ! \cdot n\left(x_{2}, p\right) ! n\left(x_{3}, p\right) !}((m-1) !)^{n}\left(1-\left(\frac{1}{2}\right)^{n\left(x_{1}, p\right)}\right) .
$$

So, $\left|M_{f}\right| \geqslant \frac{1}{2} \cdot n(n-1) \cdot((m-1) !)^{n}$. As $n \geqslant 4$ it follows that $\left|M_{f}\right|>n((m-1) !)^{n}=$ $\left|M_{u_{a}}\right|$. This contradiction completes the proof.

Remark 2 By Lemmas 2 and 5 there is an $a \in A$ such that $f\left(B_{3}\right)=\{a\}$.

Lemma 6 Let $a$ be an alternative such that $f\left(B_{3}\right)=\{a\}$. Then $f\left(B_{2}\right)=\{a\}$.

Proof. Let $p$ be a profile in $B_{2}-B_{3}$. It is sufficient to prove that $f(p)=a$. Let $x$ and $y$ be two alternatives and $S$ and $T$ be two non-empty subsets of $N$, such that $S=N(x, p), T=N(y, p)$ and $S \cup T=N$. Let $s=|S|$ and $t=|T|$, and assume $s \geqslant t$. 
First we show that, if $a \in\{x, y\}$, then $f(p)=a$. So assume that $a \in\{x, y\}$. Suppose $f(p) \neq a$. Then there is a $z \in A-\{x, y\}$, an $i \in S$, and an $i$-deviation $q$ of $p$ such that $z \ldots=q(i)$ and $\ldots f(p) \ldots a \ldots=q(i)$. Since $q \in B_{3}$, by assumption $f(q)=a$ and therefore $f$ is manipulable at $q$ by $i$ towards $p$. This contradicts Lemma 5. Hence, for all profiles $r \in B_{2}$ with $a \in \operatorname{topset}(p), f(r)=a$.

Next suppose $a \notin\{x, y\}$. First consider the case $t \geqslant 2$. Suppose $f(p) \neq a$. Let $z \in\{x, y\}-f(p)$. Since $f$ is tops-only we may assume that $z \ldots=p(i)$ and $\ldots a \ldots f(p) \ldots=p(i)$ for some $i \in N(z, p)$. Let $v$ be an $i$-deviation of $p$ such that $a \ldots=v(i)$. As topset $(v)=\{x, y, a\}$, Lemma 5 implies $f(v)=a$. Hence, $f$ is manipulable at $p$ by $i$ towards $v$. Remark 1 implies $\left|M_{f}\right| \geqslant \frac{n !}{s ! t !}((m-1) !)^{n}(1-$ $\left.\left(\frac{1}{2}\right)^{t}\right)$. So

$$
\begin{aligned}
\frac{\left|M_{f}\right|}{\left|M_{u_{a}}\right|} & \geqslant \frac{n !}{2(n-2) !} \cdot \frac{3}{4} \cdot \frac{1}{n} \\
& \geqslant \frac{3(n-1)}{8} \\
& >1,
\end{aligned}
$$

where the last inequality follows since $n \geqslant 4$. This is a contradiction and therefore $f(p)=a$.

Finally, consider the case $t=1$ (and still $a \notin\{x, y\}$ ). Suppose $f(p) \neq a$. Consider, for $i \in S$, an $i$-deviation $w$ of $p$ with $y \ldots=w(i)$ and $\ldots f(p) \ldots a \ldots=$ $w(i)$. By the previous paragraph $f(w)=a$ and therefore $f$ is manipulable at $w$ by $i$ towards $p$. By Remark 1 applied to the profile $w$ it follows that $\left|M_{f}\right| \geqslant \frac{n !}{(n-2) ! 2 !}((m-1) !)^{n}\left(1-\left(\frac{1}{2}\right)^{2}\right)$, and similarly as above this implies that $\left|M_{f}\right|>\left|M_{u_{a}}\right|$. This is a contradiction and therefore $f\left(B_{2}\right)=\{a\}$.

Lemmas 2, 5, and 6 imply that any minimally manipulable rule $f \in F$ is constant on $B_{2}$. By copying the proof of Theorem 1 we have the following result.

Theorem 2 Let $n>m \geqslant 3$. Let $f \in F$. Then $\left|M_{f}\right| \leqslant\left|M_{g}\right|$ for all $g \in F$ if and only if $f$ is a unanimity rule with status quo.

The following consequence of Theorem 2 is immediate.

Corollary 1 Let $n>m \geqslant 3$. Let $f \in F$. Then $\left|M_{f}\right| \leqslant\left|M_{g}\right|$ for all unanimous $g \in F$ if and only if $f$ is a unanimity rule with status quo.

\section{Conclusion}

In Theorem 2 we have characterized all minimally manipulable tops-only, surjective and anonymous social choice rules - hence all minimally manipulable surjective and anonymous voting rules - under the assumption that there are more agents (voters) than alternatives (candidates). Although this covers many cases of interest, it is also worthwhile to investigate the case where the number 
of agents is not larger than the number of alternatives. The combinatorial arguments used to derive the results in the preceding sections can no longer be used since they depend on the assumption $n>m$.

In the appendix we present some results for the case of two agents. It turns out, indeed, that unanimity rules with status quo are no longer per se the minimally manipulable ones among all tops-only, surjective (or even unanimous) and anonymous social choice rules. We do not have a complete characterization for this case. We do, however, have a complete characterization (for $n=2$ ) if we strengthen unanimity to Pareto optimality. An alternative is Pareto dominated in a profile of preferences if there is another alternative that is ranked higher by all agents. A choice rule is Pareto optimal if it never picks a Pareto dominated alternative.

Let $R=a_{1} a_{2} \ldots a_{m}$ be a linear ordering of the alternatives. Let the choice rule $f_{R}: L(A)^{\{1,2\}} \rightarrow A$ assign to every profile $p$ the element of topset $(p)$ which is ranked higher under $R$, i.e., the element with the lower number. Obviously, $f_{R}$ is tops-only, anonymous, and Pareto optimal. The following theorem is proved in the appendix.

Theorem 3 Let $n=2$ and $m \geqslant 3$. Let $f$ be a Pareto optimal, anonymous and tops-only choice rule such that for all Pareto-optimal, tops-only and anonymous choice rules $g$ we have $\left|M_{f}\right| \leqslant\left|M_{g}\right|$. Then $f=f_{R}$ for some linear ordering $R$ of $A$.

Pareto optimality is, obviously, preferable to unanimity and so it is worthwhile to investigate minimal manipulability under this stronger condition. This is left to future research.

\section{A Appendix: The case of two agents}

In this appendix we assume throughout that $n=2$ and $m \geqslant 3$. Note that for a unanimity rule with status quo $u_{a}$ we now have $\left|M_{u_{a}}\right|<\frac{1}{2} n(m-1)(m-$ $2)((m-1) !)^{n}$ since there are profiles which can be manipulated by both agents and therefore are counted twice in (1). In Example 1 below we establish the exact value of $\left|M_{u_{a}}\right|$.

\section{A.1 Unanimous choice rules}

Let $f$ a be unanimous, anonymous and tops-only choice rule. For an alternative $a$ define the opportunity set $O_{f}(a)=\left\{f(p): p \in L(A)^{N}, a \in \operatorname{topset}(p)\right\}$. Then

$$
\begin{aligned}
M_{f}= & \left\{p \in L(A)^{N}: \text { there are } i \in N, a \in \operatorname{topset}(p), b \in O_{f}(a)\right. \text { such that } \\
& b \neq f(p) \text { and } p(i)=\ldots b \ldots f(p) \ldots\} .
\end{aligned}
$$

For alternatives $a$ and $b$ let $M_{f}(a, b)=\left\{p \in M_{f}: \operatorname{topset}(p)=\{a, b\}\right\}$ be the set of profiles at which $f$ is manipulable and $a$ and $b$ are the top alternatives. Let $\widetilde{A}=\{\{a, b\}: a, b \in A$ and $a \neq b\}$ be the set of all non-ordered pairs of $A$. 
As $f$ is tops-only and anonymous, $\left|M_{f}\right|=\sum_{\{a, b\} \in \widetilde{A}}\left|M_{f}(a, b)\right|$. Therefore we shall have a closer look at the sets $M_{f}(a, b)$. Let $a$ and $b$ be alternatives with $a \neq b$ and consider profiles $p$ with topset $(p)=\{a, b\}$. Since $f$ is tops-only and anonymous the outcome at all these profiles is the same, say $c$. We distinguish the following situations.

(a) If $c \notin\{a, b\}$ and if $a \in O_{f}(b)$ or $b \in O_{f}(a)$, then all profiles $q$ with $\operatorname{topset}(q)=\{a, b\}$ are in $M_{f}(a, b)$, yielding $\left|M_{f}(a, b)\right|=2 \cdot((m-1) !)^{2}$. In this case set $\delta_{f}(a, b)=1$.

(b) If $c \notin\{a, b\}$ and $a \notin O_{f}(b)$ and $b \notin O_{f}(a)$, then all profiles $q$ such that $N=\{i, j\}$ and for some $x \in O_{f}(a) \backslash\{c\}$ or for some $y \in O_{f}(b) \backslash\{c\}$ with $q(i)=b \ldots x \ldots c \ldots$ or $q(j)=a \ldots y \ldots c \ldots$ are manipulable. This implies that $\left|M_{f}(a, b)\right|=2 \cdot((m-1) !)^{2} \cdot\left(1-\frac{1}{\left|O_{f}(a)\right| \cdot\left|O_{f}(b)\right|}\right)$. In this case set $\delta_{f}(a, b)=$ $1-\frac{1}{\left|O_{f}(a)\right| \cdot\left|O_{f}(b)\right|}$.

(c) If $c=a$ and $b \in O_{f}(a)$, then all profiles $q$ with $\operatorname{topset}(q)=\{a, b\}$ are in $M_{f}(a, b)$, yielding $\left|M_{f}(a, b)\right|=2 \cdot((m-1) !)^{2}$. In this case set $\delta_{f}(a, b)=1$.

(d) If $c=a$ and $b \notin O_{f}(a)$, then all profiles $q$ such that $N=\{i, j\}$ and for some $x \in O_{f}(a) \backslash\{a\}$ with $q(i)=b \ldots x \ldots c \ldots$ are manipulable. This yields that $\left|M_{f}(a, b)\right|=2 \cdot((m-1) !)^{2} \cdot\left(1-\frac{1}{\left|O_{f}(a)\right|}\right)$. In this case set $\delta_{f}(a, b)=1-\frac{1}{\left|O_{f}(a)\right|}$.

Using the notation in cases (a)-(d) we have that $\left|M_{f}\right|=2 \cdot((m-1) !)^{2} \cdot \sum_{\{a, b\} \in \widetilde{A}}$ $\delta_{f}(a, b)$. Consider a reflexive and antisymmetric relation $R^{a}$ on $A$ such that $a$ is the unique best alternative on $A$ with respect to $R^{a}$, i.e., for all alternatives $x \neq a$ we have $(a, x) \in R^{a}$ and $(x, a) \notin R^{a}$. The relation $R^{a}$ induces a unanimous anonymous and tops-only choice rule $f_{R^{a}}$, defined for all profiles $p$ with $\operatorname{topset}(p)=\{x, y\}$ as follows: $f_{R^{a}}(p)=x$ if $(x, y) \in R^{a}, f_{R^{a}}(p)=y$ if $(y, x) \in R^{a}$ and $f_{R^{a}}(p)=a$ in all other cases. Observe that the rule $f_{R}$ defined in Section 5 is a special case.

Example 1 Let $R^{a}=\{(x, y) \in A \times A: x \in\{y, a\}\}$. Then $f_{R^{a}}$ is the unanimity rule $u_{a}$. Moreover, $O_{f_{R^{a}}}(x)=\{a, x\}$ for all alternatives $x$. For alternatives $x$ and $y$ with $x \neq y$ we have $\delta_{f_{R^{a}}}(x, y)=0$ if $a \in\{x, y\}$, and by (b): $\delta_{f_{R^{a}}}(x, y)=$ $\left(1-\frac{1}{2 \cdot 2}\right)=\frac{3}{4}$ if $a \notin\{x, y\}$. This implies that $\left|M_{u_{a}}\right|=2 \cdot((m-1) !)^{2} \cdot \frac{(m-1)(m-2)}{2}$. $\frac{3}{4}=\frac{3}{4} \cdot(m-1)(m-2) \cdot((m-1) !)^{2}$.

Example 2 Let the alternatives in $A$ be numbered, say $a_{1}, a_{2}, \ldots, a_{m}$. Consider the linear ordering $R^{a}=R^{a_{1}}=a_{1} a_{2} a_{3} \ldots a_{m}$. Then the choice rule $f_{R^{a}}$ picks the lowest numbered alternative among the set of top alternatives at a profile. This is the choice rule introduced before in Section 5. Clearly $O_{f_{R^{a}}}\left(a_{t}\right)=$ $\left\{a_{1}, a_{2}, \ldots, a_{t}\right\}$ and for alternatives $a_{i}$ and $a_{j}$ with $i<j, \delta_{f_{R^{a}}}\left(a_{i}, a_{j}\right)=0$ if $a \in\left\{a_{i}, a_{j}\right\}$ and, by $(\mathrm{d}), \delta_{f_{R^{a}}}\left(a_{i}, a_{j}\right)=\left(1-\frac{1}{i}\right)$ otherwise. This implies that $\left|M_{f_{R^{a}}}\right|=2 \cdot((m-1) !)^{2} \cdot \sum_{k=2}^{m-1}(m-k) \frac{k-1}{k}$. 
Example 3 Let the alternatives be numbered as in the previous example. Consider $R^{a}=\left\{\left(a_{i}, a_{j}\right): i \leqslant j\right.$ or $\left.(i, j)=(3,2)\right\}$. So $R^{a}$ is equal to the ordering of the previous example except for the fact that $a_{2}$ is indifferent to $a_{3}$. For alternatives $a_{i}$ and $a_{j}$ with $i<j, \delta_{h_{R^{a}}}\left(a_{i}, a_{j}\right)=0$ if $a \in\left\{a_{i}, a_{j}\right\}$. By (d), $\delta_{h_{R^{a}}}\left(a_{i}, a_{j}\right)=$ $\left(1-\frac{1}{i}\right)$ if $3 \notin\{i, j\}, \delta_{h_{R^{a}}}\left(a_{i}, a_{j}\right)=\left(1-\frac{1}{2}\right)$ if $i=3$ and $\delta_{h_{R^{a}}}\left(a_{2}, a_{3}\right)=\left(1-\frac{1}{2 \cdot 2}\right)$. So $\left|M_{h_{R^{a}}}\right|=2 \cdot((m-1) !)^{2}\left(\frac{3}{4}+2(m-3) \frac{1}{2}+\sum_{k=4}^{m-1}(m-k) \frac{k-1}{k}\right)$. For later reference let this rule be denoted by $\widetilde{g}$.

Let $R$ be a linear ordering of $A$. Then for $3 \leqslant m \leqslant 12$ it follows that $\left|M_{u_{a}}\right|>$ $\left|M_{f_{R}}\right|$, but for $m>12$ it follows that $\left|M_{u_{a}}\right|<\left|M_{f_{R}}\right|$. Note also that $\left|M_{f_{R}}\right|>$ $\left|M_{\tilde{g}}\right|$ whenever $\frac{3}{4}+2(m-3) \frac{1}{2}<(m-2) \frac{1}{2}+(m-3) \frac{2}{3}$. This is precisely the case if $m \geqslant 5$. It is straightforward although tedious to show that for $n=2$ and $m \in\{3,4\},\left|M_{f_{R}}\right|=\min \left\{\left|M_{h}\right|: h\right.$ is tops-only, unanimous and anonymous $\}$, but, thus, this result does not extend to $m \geqslant 5$. For large $m$, unanimity rules with status quo $a$ might again be minimally manipulable.

\section{A.2 Pareto optimal choice rules}

In this subsection we prove Theorem 3.

Proof. By Pareto optimality, anonymity, and tops-onliness, for profiles $p$ with topset $(p)=\{a, b\}$, we have that $f(p) \in\{a, b\}$. So $f$ induces a complete and antisymmetric relation $R$ on $A$, defined for all alternatives $a$ and $b$ as follows: $(a, b) \in R$ if $f(p)=a$ for some profile $p$ with $\operatorname{topset}(p)=\{a, b\}$. Now it is straightforward to see that in calculating $\left|M_{f}(a, b)\right|$ for some $(a, b) \in R$ with $a \neq b$, only case (d) above applies, so $\left|M_{f}(a, b)\right|=2 \cdot((m-1) !)^{2} \cdot\left(1-\frac{1}{\left|O_{f}(a)\right|}\right)$. It is therefore sufficient to show that a transitive $R$ yields the smallest $\left|M_{h_{R}}\right|$. Let $a$ and $b$ be two alternatives with $a \neq b$ and $\left|O_{f}(a)\right| \leqslant\left|O_{f}(b)\right|$. It is sufficient to prove that $(a, b) \in R$. To the contrary suppose $(b, a) \in R$. Let $\bar{R}$ be equal to $R$ except for the pair $(b, a)$ which is swapped, i.e., $\bar{R}=(R \cup\{(a, b)\})-\{(b, a)\}$. We derive the contradiction $\left|M_{f}\right|>\left|M_{h_{\bar{R}}}\right|$ (which completes the proof). Now $O_{h_{\bar{R}}}(a)=O_{f}(a)-\{b\}, O_{h_{\bar{R}}}(b)=O_{f}(b) \cup\{b\},\left|O_{h_{\bar{R}}}(a)\right|=\left|O_{f}(a)\right|-1,\left|O_{h_{\bar{R}}}(b)\right|=$ $\left|O_{f}(b)\right|+1$ and

$$
\begin{aligned}
\left|M_{f}\right|-\left|M_{h_{\bar{R}}}\right|= & {\left[\sum_{(b, x) \in R, x \notin\{a, b\}}\left(1-\frac{1}{\left|O_{f}(b)\right|}\right)-\left(1-\frac{1}{\left|O_{f}(b)\right|+1}\right)\right] } \\
& +\left[\sum_{(a, x) \in R, x \neq a}\left(1-\frac{1}{\left|O_{f}(a)\right|}\right)-\left(1-\frac{1}{\left|O_{f}(a)\right|-1}\right)\right] \\
& +\left(1-\frac{1}{\left|O_{f}(b)\right|}\right)-\left(1-\frac{1}{\left|O_{f}(a)\right|-1}\right) \\
= & \left(m-\left|O_{f}(b)\right|-1\right) \cdot \frac{-1}{\left|O_{f}(b)\right| \cdot\left(\left|O_{f}(b)\right|+1\right)} \\
& +\left(m-\left|O_{f}(a)\right|\right) \cdot \frac{1}{\left|O_{f}(a)\right| \cdot\left(\left|O_{f}(a)\right|-1\right)}
\end{aligned}
$$




$$
\begin{aligned}
& +\frac{1}{\left|O_{f}(a)\right|-1}-\frac{1}{\left|O_{f}(b)\right|} \\
& >0 .
\end{aligned}
$$

The last inequality follows since $\left|O_{f}(a)\right| \leqslant\left|O_{f}(b)\right|$. So, $\left(m-\left|O_{f}(b)\right|-1\right) \leqslant$ $\left(m-\left|O_{f}(a)\right|\right), \frac{1}{\left|O_{f}(b)\right| \cdot\left(\left|O_{f}(b)\right|+1\right)} \leqslant \frac{1}{\left|O_{f}(a)\right| \cdot\left(\left|O_{f}(a)\right|-1\right)}$ and $\frac{1}{\left|O_{f}(b)\right|}<\frac{1}{\left|O_{f}(a)\right|-1}$.

\section{References}

[1] F. Aleskerov, E. Kurbanov, Degree of manipulability of social choice procedures, in: Proceedings of the Third International Meeting of the Society for the Advancement of Economic Theory, Springer-Verlag, Berlin/Heidelberg/New York, 1999.

[2] D. Black, On the rationale of group decision making, Journal of Political Economy 56 (1948), 23-34.

[3] K.C. Border, S.J. Jordan, Straightforward elections, unanimity and phantom voters, Review of Economic Studies 50 (1983), 153-170.

[4] B. Dutta, Strategic voting in a probabilistic framework, Econometrica 48 (1980), 447-456.

[5] L. Ehlers, H. Peters, T. Storcken, Threshold strategy-proofness: On manipulability in large voting systems, Games and Economic Behavior 49 (2004), 103-116.

[6] P. Fristrup, H. Keiding, Minimal manipulability and interjacency for twoperson social choice functions, Social Choice and welfare 15 (1998), 455467.

[7] A. Gibbard, Manipulation of voting schemes: A general result, Econometrica 41 (1973), 587-601.

[8] A. Gibbard, Manipulation of schemes that mix voting and chance, Econometrica 45 (1977), 665-681.

[9] A. Gibbard, Straightforwardness of game forms with lotteries as outcomes, Econometrica 46 (1978), 595-614.

[10] J.S. Kelly, Minimal manipulability and local strategy-proofness, Social Choice and Welfare 5 (1988), 81-85.

[11] J.S. Kelly, Interjacency, Social Choice and Welfare 6 (1989), 331-355.

[12] J.S. Kelly, Almost all social choice rules are highly manipulable, but a few aren't, Social choice and Welfare 10 (1993), 161-175.

[13] H.K. Kim, F.W. Roush, Non-manipulability in two dimensions, Mathematical Social Sciences 8 (1984), 29-43. 
[14] S. Maus, H. Peters, T. Storcken, Minimal manipulability: Unanimity and non-dictatorship, working paper, RM/04/006, University of Maastricht (2004a).

[15] S. Maus, H. Peters, T. Storcken, Minimal manipulability: Anonymity and surjectivity, working paper, RM/04/007, University of Maastricht (2004b).

[16] S. Maus, H. Peters, T. Storcken, Minimal manipulability: Anonymity and unanimity, working paper, RM/04/0026, University of Maastricht (2004c).

[17] H. Moulin H., On strategy-proofness and single peakedness, Public Choice 35 (1980), 437-455.

[18] P.K. Pattanaik, Counter-threats strategic manipulation under voting schemes, Review of Economic Studies 43 (1976a), 191-204.

[19] P.K. Pattanaik, Threats and counter-threats and strategic voting, Econometrica 44 (1976b), 91-104.

[20] M.A. Satterthwaite, Strategy-proofness and Arrow's conditions: Existence and correspondence theorem for voting procedures and social welfare functions, Journal of Economic Theory 10 (1975), 187-217.

[21] L. Zhou, Impossibility of strategy-proof mechanisms in economies with pure public goods, Review of Economic Studies 58 (1990), 107-119. 